\title{
Comparison of 3-D Fracture Analysis Methods Based on ANSYS Workbench
}

\author{
Dejun Liu ${ }^{a}$, Fang Xie ${ }^{b}$, and Ningbo Gao ${ }^{c}$ \\ Xi'an Hi-tech Institute, Xi'an 710025, China; \\ a28003370@qq.com, b1468786256@qq.com, cgaomoy@qq.com
}

Keywords: Pressure vessel, 3-D crack, ANSYS Workbench.

\begin{abstract}
By establishing cylinder pressure vessel with a crack model, this paper presents the similarities and differences of semi-ellipse crack method and arbitrary method of 3-D fracture analysis based on finite element software ANSYS Workbench to explore the effect for the whole structure and single-crack results of the two methods. By establishing a model with a crack, importing the model to analysis environment and analyzing results compare the two method effect for pressure vessel wholly. From stress distribution, structural deformation and stress intensity factor distribution results indicate that numerical values on structural deformation and stress intensity factor of the two methods are nearly equal and the gap on stress value is large and the stress result of semi-ellipse crack fracture method is more conservative for the structural assessment.
\end{abstract}

\section{Introduction}

For pressure equipment working under the hyperbaric environment, such as pressure vessel and pressure pipe, those equipment are easier to cause safety accidents because of equipment fracture problems which occupy large proportion among numerous safety accidents ${ }^{[1,2]}$. Fracture issues are related with structure bodies which contain cracks or defects. And causes of crack generation are various, such as manufacture or service process, welding and fatigue, any of those causes can generate cracks $^{[3-5]}$. The crack can influence the whole structure strength, and to some extent, it can grow and extend to a certain length causing the whole structure bodies damage under certain stress level ${ }^{[6]}$. Therefore, the structure analysis results should be more conservative for either designers or users and should make further assessment for equipment with cracks. If equipment with cracks is analyzed through experimental research, manpower and material resources are cost pretty high for that, so many technicians would develop the pre-analysis by the use of mechanical analysis software and the finite element method has been the mainstream method which is applied to the engineering application field. This paper mainly compares the result analysis of two 3-D crack modeling methods: semi-ellipse crack and arbitrary crack, which are contained in the finite element software ANSYS Workbench. The paper possesses some value on engineering practice application.

\section{Geological Modeling and Mesh Generation}

\subsection{The Whole Model}

The cylinder pressure vessel model is chosen to be the whole model to analyze. The length of the vessel is $1500 \mathrm{~mm}$, wall thickness is $60 \mathrm{~mm}$, and the inside radius is $500 \mathrm{~mm}$. The material of pressure vessel is the low carbon alloy steel which is $15 \mathrm{MnMoVN}$. The property of $15 \mathrm{MnMoVN}$ is that elasticity modulus is $2.15 \mathrm{e}^{\wedge} 5 \mathrm{MPa}$ and Poisson ratio is $0.3^{[7]}$. The whole model is shown as figure 1 :

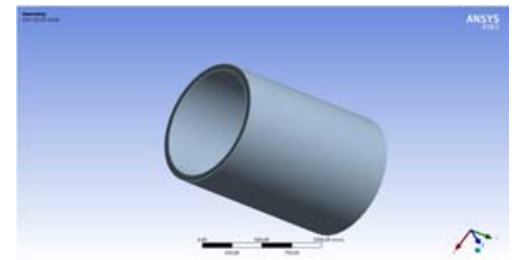

Fig. 1 Cylinder pressure vessel 


\subsection{Crack Surface Modeling}

In the previous ANSYS versions, ANSYS Workbench only provides the 3-D crack modeling of semi-ellipse crack which is inserted to the structure model without modeling independent crack model. After the whole model is meshed completely, the semi-ellipse crack can be inserted into the position directly which contains defect. Considering practical crack shapes are various, so from the later versions of ANSYS 17.0, Workbench provides another crack modeling function called arbitrary crack. Arbitrary crack is also inserted into the position containing crack, but the main distinction to semiellipse crack is that the crack surface model and whole model should be established respectively. To compare the differences between the two crack modeling methods of ANSYS Workbench which lead to the effect for results, using arbitrary crack method to establish a semi-ellipse crack model makes the size of crack is same to the crack which is modeled by semi-ellipse crack method. The half of long axis and short axis length are set $30 \mathrm{~mm}$ and $10 \mathrm{~mm}$ respectively.

For the model established by arbitrary crack method, a semi-ellipse surface body which stands for crack should be modeled in Design Modeler. The surface body would better to be established on the position containing defect in the modeling environment directly. As is figure 2 shown:

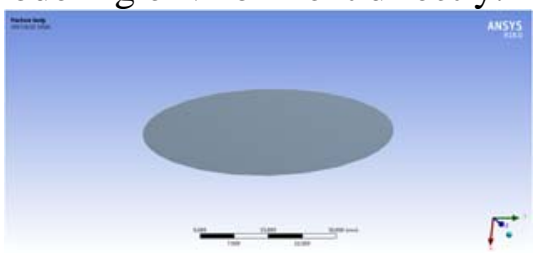

Fig 2. Semi-ellipse surface crack body

In the analysis environment, no matter which crack modeling method is used, to ensure the exact position of crack surface body and define the crack tip and crack growth direction, a local coordinate system ought to be set before analysis. The $\mathrm{X}$ axis of the local coordinate system should point to crack growth direction or crack tip and Y axis should be perpendicular to the crack surface. Because of using contour integral method to calculate relevant property parameters of crack, the number of contours and crack tip elements should be pointed approximately to improve computational accuracy so that the crack can be inserted into the whole structure body successfully.

\subsection{Mesh Generation}

Because of the existence of crack tip singularity, the usual elements surrounding the crack tip should be generated refined mesh in order to derive reliable stress intensity factor results. ANSYS requires the part structure body with cracks should be meshed with tetrahedron elements, while other bodies without cracks can be set arbitrary element types. Meanwhile, the crack mesh generation settings of Workbench are more convenient than settings under ANSYS classic analysis environment, because under Workbench environment mesh refinement of the crack tip is automatically to generate after accomplishment of mesh generation for the whole body ${ }^{[8-10]}$. As figure 3 shows, the number of the whole structure element is 160950, while number of total nodes is 279397.

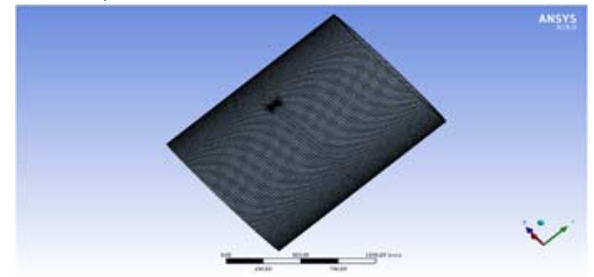

Fig. 3 Mesh Generation Model

Through yhe comparison of mesh generation surrounding the crack tip between the two methods as figure 4 shows, the mesh generation of two crack modeling indicates that the two kinds of automatically mesh generation surrounding the crack tip are approximate. But the mesh generated between two crack tips using conventional semi-ellipse crack method is more regular than arbitrary crack method. For the arbitrary crack method, the part of surface body which is inserted into structural body would be seen as crack surface body independently and further to generate relevant mesh along the surface body margin regarded as the crack tip. 

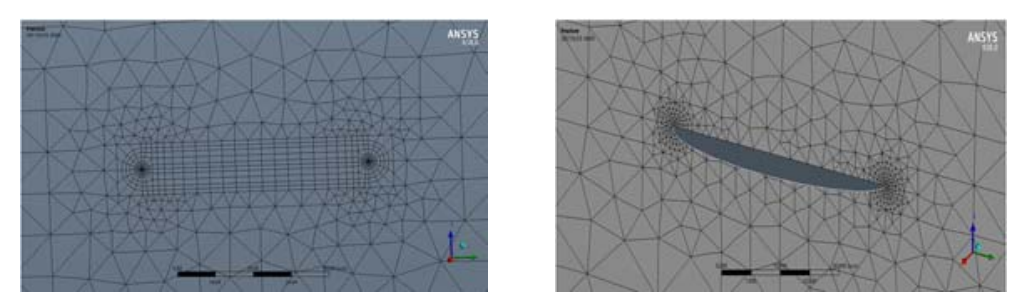

(a) Semi-ellipse crack mesh generation(b) Arbitrary crack mesh generation

Fig.4 Crack mesh generation of two methods

\subsection{Finite Element Analysis Results}

According to the practical working pressure range of pressure vessel, setting three groups of various inner pressures are $5 \mathrm{MPa}, 10 \mathrm{MPa}$ and $15 \mathrm{Mpa}$ respectively in order to compare two crack analysis methods' effect for results and analyze for assessment.
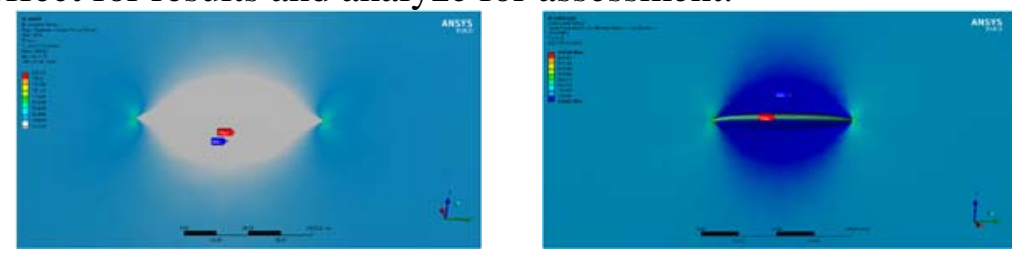

(a) Semi-ellipse crack model under 5Mpa

(b) Arrbitrary crack model under 5MPa
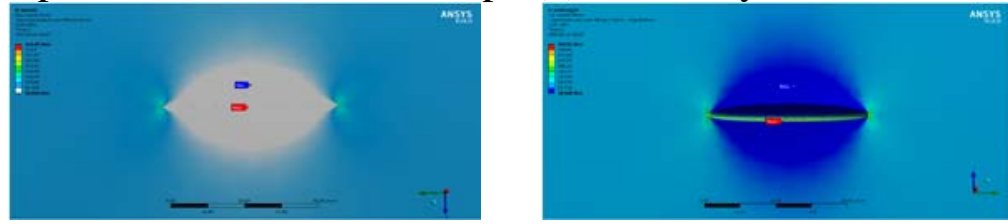

(c) Semi-ellipse crack model under 10Mpa

(d) Arrbitrary crack model under 10MPa
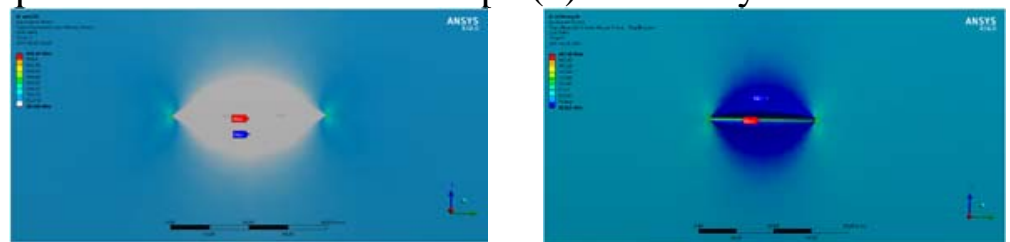

(e) Semi-ellipse crack model under 15MPa (f) Arbitrary crack model under 15MPa

Fig. 5 Stress distribution under various working pressure

As figure 5 shows, no matter using which method modeling the crack, the total maximum stress position distributes on the deepest location of the crack tip and minimum stress position distributes on the opening location of the crack two sides. But as the load increases, the stress value gap of using two crack modeling methods is more and more large. The stress values on the above working pressures under using semi-ellipse crack method are 208MPa, 417MPa, and 625MPa respectively. While the stress values on the above working pressures under using arbitrary crack method are $174 \mathrm{MPa}, 349 \mathrm{MPa}$ and $467 \mathrm{MPa}$. From the numerical values comparison of two crack modeling crack, it finds that on the same conditions stress values calculated from semi-ellipse crack method are much higher than stress magnitudes calculated from arbitrary crack method. In other words, the plastic deformation position of would appears much earlier than the crack modeled by arbitrary method. And the load value leads to plastic deformation for pressure vessel crack modeled by semi-ellipse method would be lower than the load value needed by arbitrary crack model causing plastic deformation. It is known from the consequence that the total stress analysis results for pressure vessel got from the crack modeled by semi-ellipse crack method would be much more conservative than the model established by arbitrary crack method. As a result, if the practical crack can be simplified to a semiellipse crack, considering the safety factors and effects, using semi-ellipse crack method to assess and evaluate pressure vessel properties would be more conservative than results of arbitrary crack method.

From figure 6, it shows that the deformation of pressure vessel with cracks using two crack modeling methods is similar under various working pressures. The minimum deformation position locates on the bottom of the vessel where is constrained and the deformation magnitude of the vessel is becoming high from vessel bottom to top. Combining deformation values, the total deformation 
magnitude of pressure vessel under semi-ellipse crack method and arbitrary crack method modeling is equal. And maximum deformation values under various working pressures are $0.13 \mathrm{~mm}, 0.26 \mathrm{~mm}$ and $0.39 \mathrm{~mm}$ respectively. Thus, if total deformation of pressure vessels is discussed, there is no difference for the model established by two crack modeling methods under various pressures.
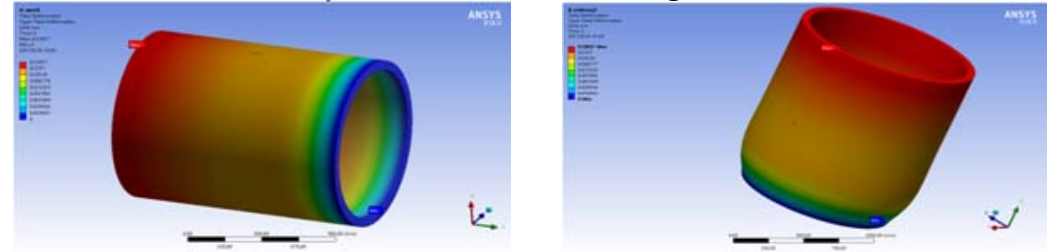

(a) Semi-ellipse crack model under 5MPa

(b) Arbitrary crack model under $5 \mathrm{MPa}$
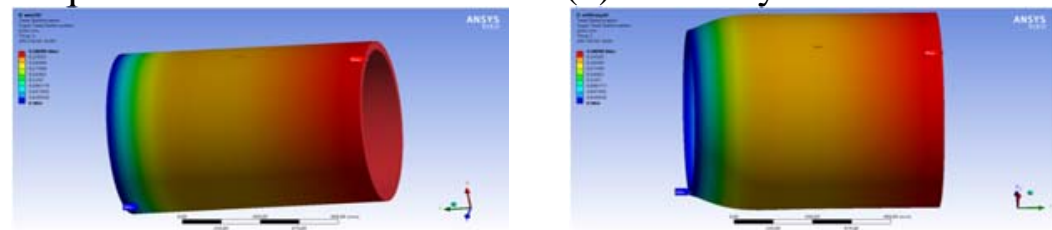

(c) Semi-ellipse crack model under 10MPa(d) Arbitrary crack model under10MPa
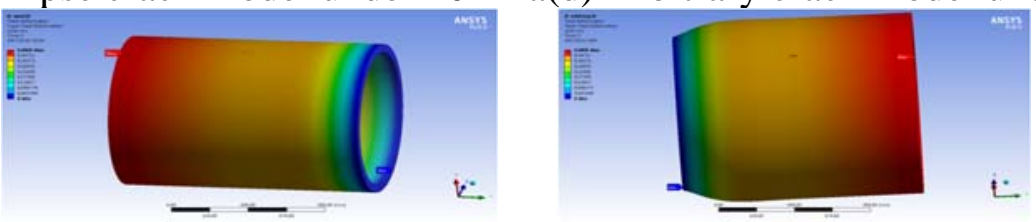

(e) Semi-ellipse crack model under 15Mpa (f) Arbitrary crack model under 15MPa

Fig. 6 Deformation distribution under various working pressure

Because there exists stress singular point on the crack tip and ANSYS uses contour integral method to calculate stress intensity factor, those would influence solution accuracy. So it is convenient to choose results got from third to sixth contour. This solution uses sixth contour's results to analyze. As figure 7 shows, stress intensity factor distribution using two crack modeling methods under various working pressure is almost same. The maximum stress intensity factor locates on the deepest position locating on the crack tip. It indicates that this position would reach to the critical stress intensity factor first under exerting pressures, which means the crack growth would from that position. From total stress intensity factor distribution of crack tips, the value of stress intensity factor increases from the tip's two sides to middle. That means the middle position of the crack tip margin is easier to occur crack growth. The stress intensity factors of the crack modeled by semi-ellipse crack method under various loads are 223.96MPa $\bullet \mathrm{mm}^{1 / 2}, 447.92 \mathrm{MPa} \cdot \mathrm{mm}^{1 / 2}$, and $671.88 \mathrm{MPa} \cdot \mathrm{mm}^{1 / 2}$.

While the stress intensity factors of the crack modeled by arbitrary crack method under various loads are $224.14 \mathrm{MPa} \cdot \mathrm{mm}^{1 / 2}, 448.29 \mathrm{MPa} \cdot \mathrm{mm}^{1 / 2}$, and $672.58 \mathrm{MPa} \cdot \mathrm{mm}^{1 / 2}$.From the numerical comparison of stress intensity factor, values are almost same and the gap is quite small. It indicates that the influence for the crack tip of defect modeled by two crack modeling methods is same on the same load and boundary conditions. If the crack analysis is independently researched, the two fracture methods provided by Workbench are both applicative.
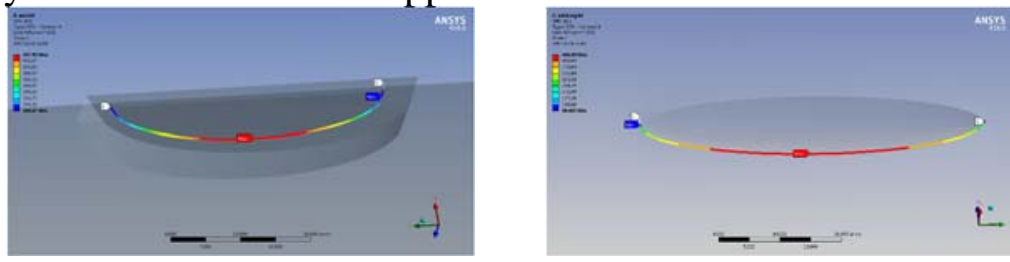

(c) Semi-ellipse crack model under 10MPa

(d) Arbitrary crack model under 10MPa
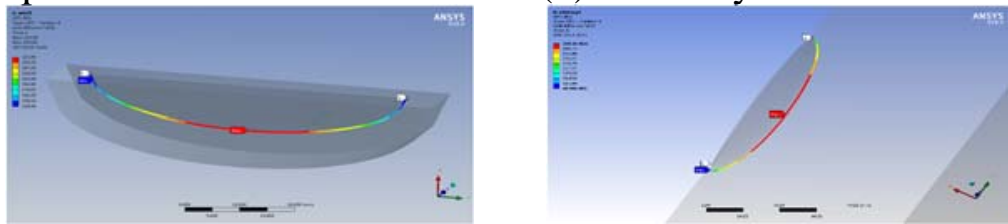

(a) Semi-ellipse crack model under 5Mpa (b) Arbitrary crack model under 5MPa 


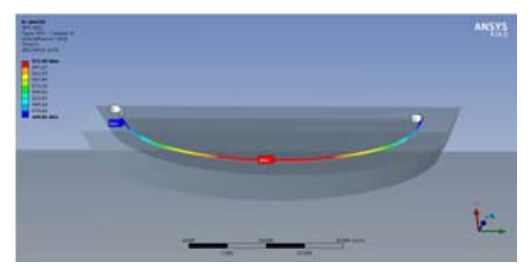

(e) Semi-ellipse crack model under 15MPa

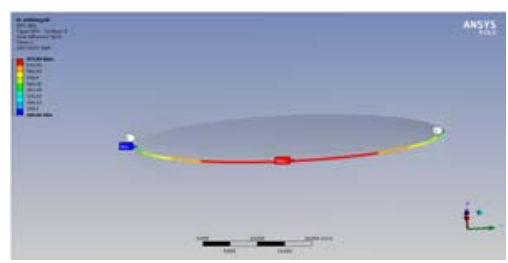

(f) Arbitrary crack model under 15MPa

Fig.7 Stress intensity factors distribution under various working pressure

\section{Summary}

(1) Based on finite element software ANSYS Workbench, This paper has compared two 3-D crack analysis methods contained by Workbench, semi-ellipse crack method and arbitrary crack method. From modeling, setting boundary conditions and result treatment processes, the final comparison has clarified the differences between the above two methods.

(2) Through modeling the same size semi-ellipse crack modeled by two fracture analysis methods independently, making the finite element analysis can be compared together. The results indicate that stress values got from semi-ellipse crack method are higher than those got from arbitrary crack method so that semi-ellipse crack method's results are more conservative for pressure vessel assessment and evaluation. While total deformation and stress intensity factor values got from the above two crack methods are almost equal. Thus, if structural deformation and independent crack stress intensity factor considered only, results derived from semi-ellipse crack method and arbitrary would be applicable to practical application.

(3) This paper only compares 3-D crack analysis results based on Workbench, while work for 2D crack analysis methods would develop further in the future.

\section{References}

[1]. Yuguang Cao, Qian Kong, Kai Tian, et al. Calculation of stress intensity factors of axial cracks on pressure pipelines using FEM simulation[J]. Oil Field Equipment, 2010(08): 1-7.

[2]. Lianhua Zhao. Analysis on three-dimensional situation based ANSYS for plate crack tip on load[J]. Metallurgical Equipment, 2007(S1): 4-5.

[3]. Jing Shao, Deqi Zhang, Lulu Fu, et al. Fracture mechanics of elliptical crack on pipe surface with finite element analysis[J]. Contemporary Chemical Industry, 2015(08): 1972-1973.

[4]. Yang Chen, Zhimin Lu, Jianlei Qi, et al. Three dimensional finite element analysis of a defect containg buffer[J]. Journal of Inner Mongolia University of Technology, 2016, 35(3): 189-193.

[5]. Jianlei Qi, Zhimin Lu, Fei Zhao, et al. Safety assessment for pressure vessel with crack and solution by using intensity factor[J]. Process Equipment and Piping, 2016(03): 21-23.

[6]. Nan Jiang, Zhaofeng Li. Calculation and analysis of fracture parameters of pressure vessels surface crack with finite element method[J]. Industrial Safety and Environmetnal Protection, 2011(09): 58-60.

[7]. Honggao Geng, Zhongbao Qin, Zhiqing Guo, et al. Study on finite element analysis and stress assessment of the spherical pressure vessel[J]. Piezoelectrics and Acoustooptics, 2016(10): 1618.

[8]. Jiasheng He, Mengqi Yan. Stress analysis and pipe the study of stress intensity factor for pressure with longitudinal and inner crack[J]. Chemical Equipment Technology, 2017(01): 1-4.

[9]. Xu Jia, Xuteng $\mathrm{Hu}$, Yingdong Song. Calculation formula for stress intensity factors of CT specimens based on three dimensional finite element solutions[J]. Metarials for Mechnical Engineering,2015(12): 30-34.

[10]. Xiaohai Wu, Li Tian, Song Zhou, et al. The study in stress intensity factor of crack tip based on ANSYS [J]. Mechanical Engineering, 2011(01): 42-43. 